\title{
Classification of toric log del Pezzo surfaces with few singular points
}

\author{
Yusuke Suyama* \\ Department of Mathematics \\ Graduate School of Science \\ Osaka University \\ Osaka, Japan \\ y-suyama@cr.math.sci.osaka-u.ac.jp
}

Submitted: Oct 12, 2019; Accepted: Feb 2, 2021; Published: Feb 12, 2021

(C) The author. Released under the CC BY-ND license (International 4.0).

\begin{abstract}
We give a classification of toric log del Pezzo surfaces with two or three singular points. Our proofs are purely combinatorial, relying on the bijection between toric log del Pezzo surfaces and the so-called LDP-polygons introduced by Dais and Nill.
\end{abstract}

Mathematics Subject Classifications: 14M25, 14Q10, 52B20

\section{Introduction}

A normal projective surface is called log del Pezzo surface if it has at worst log-terminal singularities (that is, quotient singularities) and its anticanonical divisor is a $\mathbb{Q}$-Cartier ample divisor. Log del Pezzo surfaces have been extensively studied and many results are known (for example [13, 14, 15, 1, 12, 9]).

When they are toric, log del Pezzo surfaces are known to be in bijection to the socalled LDP-polygons, introduced by Dais and Nill [8]. This makes their classification be a purely combinatorial problem. Let us explain this connection from the point of view of the polygon, skipping all technical algebraic-geometric details.

An LDP-polygon is a convex lattice polygon with the origin in its interior and with the property that all vertices are primitive lattice points; that is, they are of the form $(p, q) \in \mathbb{Z}^{2}$ with $\operatorname{gcd}(p, q)=1$. The bijection sends isomorphism classes of toric log del Pezzo surfaces to equivalence classes of LDP-polygons, where two LDP-polygons $Q$ and $Q^{\prime}$ are called equivalent if there is an automorphism of the lattice $\mathbb{Z}^{2}$ sending $Q$ to $Q^{\prime}$. Remember that an automorphism of $\mathbb{Z}^{2}$ is (the restriction to $\mathbb{Z}^{2}$ of) a linear map

*Supported by JSPS KAKENHI Grant Number JP18J00022. 
of determinant \pm 1 and with integer coefficients. See Section 2, in particular Corollary 8, for more details. Moreover, several algebraic-geometric invariants of a toric log del Pezzo surface $X_{Q}$ admit an easy description in terms of the corresponding LDP-polygon $Q$. Among them:

Picard number: The Picard number of $X_{Q}$ equals the number of edges (equivalently, of vertices) of $Q$ minus two. That is, triangles, quadrilaterals, and pentagons correspond respectively to Picard number $1,2,3$, etc.

Singular points: Each torus-invariant point of $X_{Q}$ corresponds to an edge of $Q$, and those points are the only possible singularities in a toric surface. Since the number and distribution of such singular points around the polygon plays an important role in this paper, we call an edge of $Q$ singular or nonsingular accordingly.

Proposition 1 ([4, Theorem 1.3.12]). An edge of an LDP-polygon $Q$ is nonsingular (that is, the corresponding torus-fixed point of the associated toric surface $X_{Q}$ is nonsingular) if and only if its two vertices form a basis of $\mathbb{Z}^{2}$. That is, if and only if $|p s-r q|=1$, where $(p, q)$ and $(r, s)$ are the vertex coordinates.

It is well known that there are exactly five nonsingular LDP-polygons (that is, five nonsingular toric del Pezzo surfaces). They are the polars of the five smooth reflexive polygons, and they are depicted in Figure 1.

Blow-ups: Suppose that $v_{i} v_{i+1}$ is a nonsingular edge in an LDP-polygon $Q$, and let $Q^{\prime}$ be the convex hull of $Q \cup\left\{v_{i}+v_{i+1}\right\}$. If $v_{i}$ and $v_{i+1}$ are still vertices of $Q^{\prime}$, then $X_{Q^{\prime}}$ is the blow-up of $X_{Q}$ at the corresponding nonsingular point. In this situation we say that the LDP-polygon $Q^{\prime}$ is a blow-up of $Q$ at the nonsingular edge $v_{i} v_{i+1}$. Blow-ups make sense algebraically even if $v_{i}$ or $v_{i+1}$ are not vertices of $Q^{\prime}$ anymore but in this case the resulting toric surface is no longer log del Pezzo. Thus, we do not consider it.

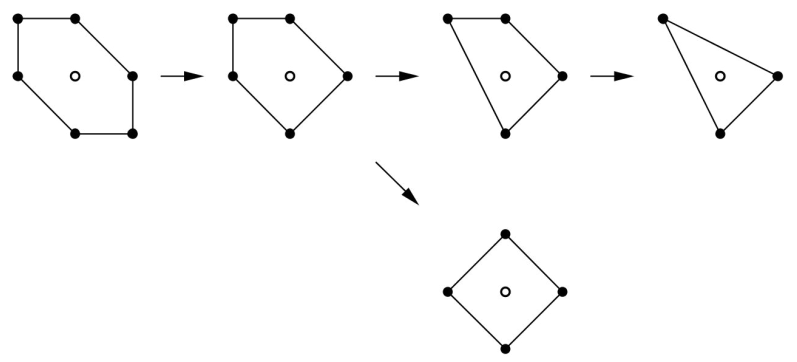

Figure 1: The five nonsingular LDP-polygons, polars of the smooth reflexive polygons. The interior dot in each polygon is the origin and arrows represent blow-ups. As customary in algebraic geometry, blow-ups are shown as reverse arrows, since there is a morphism from the blown-up variety to the original one. 
Index: The index of $X_{Q}$ is the minimum $k \in \mathbb{N}$ such that $Q$ can be described by linear inequalities of the form $a_{1} x \leqslant k, \ldots, a_{d} x \leqslant k$, with $a_{1}, \ldots, a_{d} \in \mathbb{Z}^{2}$. Put differently, it is the minimum $k \in \mathbb{N}$ such that $k Q^{\vee}$ is a lattice polygon, where $Q^{\vee}$ is the polar polygon of $Q$. For example, an LDP-polygon has index 1 if and only if its polar is again a lattice polygon. Lattice polytopes (in arbitrary dimension) whose polar is again a lattice polytope are called reflexive, and there are exactly sixteen of them in dimension two; that is, there are sixteen LDP-polygons of index 1. They include the five nonsingular ones (see [4, Theorem 8.3.7]).

It is known that there are finitely many toric log del Pezzo surfaces of any fixed index (see [10, Corollary 4.5]). Dais [5, 6] classified toric log del Pezzo surfaces of Picard number one (that is, LDP-triangles) and of index at most three, and Kasprzyk-Kreuzer-Nill [10] gave two independent algorithms that enumerate all toric log del Pezzo surfaces up to any given index.

In this paper, we focus on the number of singular points on toric log del Pezzo surfaces. Partial results on this exist even in the general, perhaps non-toric case: Belousov $[2,3]$ proved that a log del Pezzo surface of Picard number one has at most four singular points, and Kojima [11] classified log del Pezzo surfaces of Picard number one with unique singular points. Dais has recently classified all toric log del Pezzo surfaces with unique singular points:

Theorem 2 ([7, Theorem 1.4]). An LDP-polygon has exactly one singular edge if and only if it is equivalent to one of the following:

(1) $\operatorname{conv}\left\{\left(\begin{array}{c}1 \\ -1\end{array}\right),\left(\begin{array}{c}p \\ 1\end{array}\right),\left(\begin{array}{c}-1 \\ 0\end{array}\right)\right\}$ for some positive integer $p$.

(2) A quadrilateral or pentagon obtained by blowing up one or both of the nonsingular edges of an LDP-triangle in part (1).

The purpose of this paper is to extend this classification for LDP-polygons with two or three singular points:

Theorem 3. An LDP-polygon has exactly two singular edges if and only if it is equivalent to one of the following:

(1) $\operatorname{conv}\left\{\left(\begin{array}{l}1 \\ 0\end{array}\right),\left(\begin{array}{l}0 \\ 1\end{array}\right),\left(\begin{array}{l}-p \\ -q\end{array}\right)\right\}$ for some $p, q \in \mathbb{Z}$ with $p, q \geqslant 2$ and $\operatorname{gcd}(p, q)=1$.

(2) $\operatorname{conv}\left\{\left(\begin{array}{l}1 \\ 0\end{array}\right),\left(\begin{array}{l}0 \\ 1\end{array}\right),\left(\begin{array}{c}-1 \\ p\end{array}\right),\left(\begin{array}{l}q \\ r\end{array}\right)\right\}$ for some $p, q, r \in \mathbb{Z}$ with

$$
p \leqslant 1, r \leqslant \min \{-p q-2,-2,-q-1, q-p q-1\}
$$

and $\operatorname{gcd}(q, r)=1$

(3) $\operatorname{conv}\left\{\left(\begin{array}{l}1 \\ 0\end{array}\right),\left(\begin{array}{l}0 \\ 1\end{array}\right),\left(\begin{array}{c}-1 \\ p+1\end{array}\right),\left(\begin{array}{c}-1 \\ p\end{array}\right),\left(\begin{array}{l}q \\ r\end{array}\right)\right\}$ for some $p, q, r \in \mathbb{Z}$ with $p \leqslant$ $0,1 \leqslant q \leqslant-r-1$ and $\operatorname{gcd}(q, r)=1$. 
Note that part (3) in Theorem 3 is the blow-up of an LDP-quadrilateral in part (2) at a nonsingular edge.

Theorem 4. An LDP-polygon has exactly three singular edges if and only if it is equivalent to one of the following:

(1) $\operatorname{conv}\left\{\left(\begin{array}{l}1 \\ 0\end{array}\right),\left(\begin{array}{l}p \\ q\end{array}\right),\left(\begin{array}{l}r \\ s\end{array}\right)\right\}$ for some $p, q, r, s \in \mathbb{Z}$ with $q \geqslant 2, s \leqslant-2, p s-q r \geqslant 2$ and $\operatorname{gcd}(p, q)=\operatorname{gcd}(r, s)=1$.

(2) $\operatorname{conv}\left\{\left(\begin{array}{l}1 \\ 0\end{array}\right),\left(\begin{array}{l}0 \\ 1\end{array}\right),\left(\begin{array}{l}p \\ q\end{array}\right),\left(\begin{array}{l}r \\ s\end{array}\right)\right\}$ for some $p, q, r, s \in \mathbb{Z}$ with

$$
p \leqslant \min \{-2,-q\}, s \leqslant \min \{-2,-r\}, p s-q r \geqslant \max \{2, p-r+1, s-q+1\}
$$

and $\operatorname{gcd}(p, q)=\operatorname{gcd}(r, s)=1$.

(3) $\operatorname{conv}\left\{\left(\begin{array}{l}1 \\ 0\end{array}\right),\left(\begin{array}{l}0 \\ 1\end{array}\right),\left(\begin{array}{c}-1 \\ p\end{array}\right),\left(\begin{array}{c}-q \\ p q-r\end{array}\right),\left(\begin{array}{c}s \\ -t\end{array}\right)\right\}$ for some

$$
p, q, r, s, t \in \mathbb{Z}
$$

with

$$
\begin{aligned}
p & \leqslant 1, \\
r & \geqslant \max \{2, q+1\}, \\
t & \geqslant \max \{2, s+1\}, \\
q t+r s-p q s & \geqslant \max \{2, t-r-p s+1, r-t-p q+1\}, \\
p q-r & \leqslant-1
\end{aligned}
$$

and $\operatorname{gcd}(q, r)=\operatorname{gcd}(s, t)=1$.

(4) The blow-up of an LDP-pentagon in part (3) at a nonsingular edge.

Our proofs of Theorems 3 and 4 use the following general properties of LDP-polygons:

Theorem 5. Let $Q$ be an LDP-polygon with at least one singular edge. Then:

(1) The nonsingular edges of $Q$ (and hence also the singular ones) form a consecutive sequence. (See Proposition 16).

(2) If $Q$ has at least two singular edges, then it has at most three nonsingular edges. (See Corollary 17).

(3) If $Q$ is not a blow-up of a smaller LDP-polygon at a nonsingular edge, then $Q$ has at most two nonsingular edges. (See Lemma 11). 
Theorems 2 and 5(2) imply that any LDP-polygon $Q$ has at most four nonsingular edges except if $Q$ is the polar of the unique smooth reflexive pentagon or hexagon. Furthermore, Theorem 5(3) implies that any LDP-polygon $Q$ with more than two nonsingular edges is a blow-up of a smaller LDP-polygon except if $Q$ is equivalent to

$$
\operatorname{conv}\left\{\left(\begin{array}{l}
1 \\
0
\end{array}\right),\left(\begin{array}{l}
0 \\
1
\end{array}\right),\left(\begin{array}{l}
-1 \\
-1
\end{array}\right)\right\} \text { or conv }\left\{\left(\begin{array}{l}
1 \\
0
\end{array}\right),\left(\begin{array}{l}
0 \\
1
\end{array}\right),\left(\begin{array}{c}
-1 \\
0
\end{array}\right),\left(\begin{array}{c}
0 \\
-1
\end{array}\right)\right\} \text {. }
$$

The structure of the paper is as follows: In Section 2, we recall the bijection between toric log del Pezzo surfaces and LDP-polygons, and we fix some notation. In Sections 3 and 4, we prove Theorems 3 and 4, respectively. The three parts of Theorem 5 are proved as we go along.

\section{Toric log del Pezzo surfaces and LDP-polygons}

We fix a notation and recall some basic facts from toric geometry which will be used in this paper. (See [4] for details.) Let $\Delta$ be a complete fan in $\mathbb{R}^{2}$. We list the primitive generators of rays, that is, one-dimensional cones, in $\Delta$ as $v_{1}, \ldots, v_{d}$ in counterclockwise order around the origin in $\mathbb{R}^{2}$, and we define $v_{0}=v_{d}$ and $v_{d+1}=v_{1}$. For $i=1, \ldots, d$, we write $v_{i}=\left(\begin{array}{c}x_{i} \\ y_{i}\end{array}\right)$. Observe that a complete fan $\Delta$ in $\mathbb{R}^{2}$ is completely described by its set of primitive ray generators $v_{1}, \ldots, v_{d}$, and that any such set defines a complete fan as long as all the $v_{i}$ are primitive (that is, $\operatorname{gcd}\left(x_{i}, y_{i}\right)=1$ ), no two are positive multiples of one another, and every open half-plane contains at least one $v_{i}$. We denote by $X(\Delta)$ the associated complete toric surface. Since $\Delta$ is simplicial, the Picard number $\rho(X(\Delta))$ of $X(\Delta)$ equals $d-2$.

Definition 6. Let $\Delta$ be a complete fan in $\mathbb{R}^{2}$. We define the map $f:\{1, \ldots, d\} \rightarrow \mathbb{Z}$ by

$$
f(i)=\operatorname{det}\left(v_{i-1}, v_{i}\right)+\operatorname{det}\left(v_{i}, v_{i+1}\right)+\operatorname{det}\left(v_{i+1}, v_{i-1}\right) .
$$

The equivalence of parts (1) and (2) in the following statement is Remark 6.7 in [5] (see also Exercise 8.3.9 in [4]). The equivalence of (2) and (3) is elementary.

Proposition 7. Let $\Delta$ be a complete fan in $\mathbb{R}^{2}$ and let $v_{1}, \ldots, v_{d}$ be its primitive ray generators, in counterclockwise order. Then, the following are equivalent:

(1) The toric surface $X(\Delta)$ is log del Pezzo.

(2) Every $v_{i}$ is a vertex of $\operatorname{conv}\left\{v_{1}, \ldots, v_{d}\right\}$.

(3) $f(i)>0$ for every $i$.

Let $Q \subset \mathbb{R}^{2}$ be an LDP-polygon and let $v_{1}, \ldots, v_{d}$ be its vertices, in counterclockwise order. We denote by $\operatorname{Sing}(Q)$ the set of singular edges of $Q$ and denote by $F_{i}$ the edge $v_{i} v_{i+1}$ of $Q$ for $i=1, \ldots, d$. For each edge $F_{i}$, we define the rational strongly convex polyhedral cone $\sigma_{i}=\left\{\lambda x \mid \lambda \geqslant 0, x \in F_{i}\right\} \subset \mathbb{R}^{2}$. Then the set $\Delta_{Q}$ of all such cones and their faces forms a complete fan in $\mathbb{R}^{2}$ whose primitive ray generators are precisely the vertices of $Q$. We define $X_{Q}$ to be the associated toric surface $X\left(\Delta_{Q}\right)$. 
Corollary 8 ([7, Proposition 4.2]). The correspondence $Q \mapsto X_{Q}$ induces a bijection between equivalence classes of LDP-polygons and isomorphism classes of toric log del Pezzo surfaces.

Example 9. Let $p$ be a positive integer and let

$$
v_{1}=\left(\begin{array}{l}
1 \\
0
\end{array}\right), v_{2}=\left(\begin{array}{l}
0 \\
1
\end{array}\right), v_{3}=\left(\begin{array}{l}
-1 \\
-p
\end{array}\right) .
$$

Then the convex hull $Q=\operatorname{conv}\left\{v_{1}, v_{2}, v_{3}\right\}$ is an LDP-triangle. The fan $\Delta_{Q}$ consists of the cones

$$
\mathbb{R}_{\geqslant 0} v_{1}+\mathbb{R}_{\geqslant 0} v_{2}, \mathbb{R}_{\geqslant 0} v_{2}+\mathbb{R}_{\geqslant 0} v_{3}, \mathbb{R}_{\geqslant 0} v_{3}+\mathbb{R}_{\geqslant 0} v_{1}
$$

and their faces. The associated toric log del Pezzo surface $X_{Q}$ is the weighted projective plane $\mathbb{P}(1,1, p)$ (see [4, Example 3.1.17]).

\section{Proof of Theorem 3}

In this section, we give a proof of Theorem 3. We will use the notation introduced in Section 2 freely. The following lemmas play key roles in the proof of Theorems 3 and 4 .

Lemma 10. Let $Q$ be an LDP-polygon with $d \geqslant 4$ edges. Suppose that there exists $i$ with $1 \leqslant i \leqslant d$ such that $F_{i-1}$ and $F_{i+1}$ are singular while $F_{i}$ is nonsingular. Then the following hold:

(1) We have $\operatorname{det}\left(v_{i+2}, v_{i-1}\right) \geqslant 2$. In particular, the cones $\sigma_{i-1}, \sigma_{i}, \sigma_{i+1}$ cover more than a half-plane.

(2) Q has at least three singular edges.

Proof. Without loss of generality, we may assume that $i=2, v_{2}=\left(\begin{array}{c}-1 \\ 0\end{array}\right), v_{3}=\left(\begin{array}{c}0 \\ -1\end{array}\right)$. Then $y_{1} \geqslant 2$ and $x_{4} \geqslant 2$.

(1) Proposition 7 and $f(2)=x_{1}+y_{1}+1$ imply $x_{1} \geqslant-y_{1}$. Since $y_{1} \geqslant 2$ and $v_{1}=\left(\begin{array}{l}x_{1} \\ y_{1}\end{array}\right)$ is primitive, we have $x_{1} \geqslant 1-y_{1}$ and $x_{1} \neq 0$. A similar argument shows that $y_{4} \geqslant 1-x_{4}$ and $y_{4} \neq 0$.

Case 1. Suppose that $x_{1} \leqslant-1$ or $y_{4} \leqslant-1$. We may assume that $x_{1} \leqslant-1$. Then $y_{4} \geqslant 1-x_{4}$ implies $x_{1} y_{4} \leqslant x_{1}\left(1-x_{4}\right)$. Since $1-x_{4} \leqslant 1-2=-1$ and $x_{1} \geqslant 1-y_{1}$, we have $x_{1}\left(1-x_{4}\right) \leqslant\left(1-x_{4}\right)\left(1-y_{1}\right)$. Thus, $x_{1} y_{4} \leqslant\left(1-x_{4}\right)\left(1-y_{1}\right)$. Therefore,

$$
\operatorname{det}\left(v_{4}, v_{1}\right)=x_{4} y_{1}-x_{1} y_{4} \geqslant x_{4} y_{1}-\left(1-x_{4}\right)\left(1-y_{1}\right)=x_{4}+y_{1}-1 \geqslant 3 \text {. }
$$

Case 2. Suppose that $x_{1} \geqslant 1$ and $y_{4} \geqslant 1$. Then both $v_{4}$ and $v_{1}$ are in

$$
\left\{\left(\begin{array}{l}
x \\
y
\end{array}\right) \in \mathbb{R}^{2} ; x, y \geqslant 0\right\} \text {. }
$$


Since $v_{1}, \ldots, v_{d}$ are arranged in counterclockwise order, we must have $\operatorname{det}\left(v_{4}, v_{1}\right)=x_{4} y_{1}-$ $x_{1} y_{4} \geqslant 1$. To deduce a contradiction we assume that $\operatorname{det}\left(v_{4}, v_{1}\right)=1$. We see that $\left(x_{4}+1\right) / x_{1}>0$ and

$$
\begin{aligned}
& \frac{x_{4}+1}{x_{1}}\left(\left(1-\frac{x_{4}}{x_{4}+1}\right) v_{3}+\frac{x_{4}}{x_{4}+1} v_{1}\right) \\
& =\frac{1}{x_{1}}\left(\begin{array}{c}
x_{1} x_{4} \\
-1+x_{4} y_{1}
\end{array}\right)=\frac{1}{x_{1}}\left(\begin{array}{c}
x_{1} x_{4} \\
x_{1} y_{4}
\end{array}\right)=v_{4} .
\end{aligned}
$$

Since $v_{4}$ is a vertex of $Q$, we must have $\left(x_{4}+1\right) / x_{1}>1$, so $x_{4} \geqslant x_{1}$. The assumption $\operatorname{det}\left(v_{4}, v_{1}\right)=1$ and $x_{4} \geqslant 2$ imply $x_{4}-1 \geqslant x_{1}$. A similar argument shows that $y_{1}-1 \geqslant y_{4}$. Thus, $x_{1} y_{4} \leqslant\left(x_{4}-1\right)\left(y_{1}-1\right)$. Therefore,

$$
1=\operatorname{det}\left(v_{4}, v_{1}\right)=x_{4} y_{1}-x_{1} y_{4} \geqslant x_{4} y_{1}-\left(x_{4}-1\right)\left(y_{1}-1\right)=x_{4}+y_{1}-1 \geqslant 3,
$$

which is a contradiction.

In every case, we obtain $\operatorname{det}\left(v_{4}, v_{1}\right) \geqslant 2$.

(2) If $d=4$, then $F_{4}$ is a singular edge by (1). Assume $d \geqslant 5$. Then by (1), there exists a lattice point $v \in\left(\operatorname{conv}\left\{0, v_{4}, v_{1}\right\} \cap \mathbb{Z}^{2}\right) \backslash\left\{0, v_{4}, v_{1}\right\}$. Since

$$
\bigcup_{j=4}^{d} \operatorname{conv}\left\{0, v_{j}, v_{j+1}\right\} \supsetneq \operatorname{conv}\left\{0, v_{4}, v_{1}\right\},
$$

there exists $j \in\{4, \ldots, d\}$ such that $v$ is an interior point of $\operatorname{conv}\left\{0, v_{j}, v_{j+1}\right\}$. In particular, $F_{j}$ is a singular edge and thus $|\operatorname{Sing}(Q)| \geqslant 3$.

Lemma 11. Let $Q$ be an LDP-polygon with at least one singular edge. Suppose that $Q$ cannot be obtained by blowing up a nonsingular edge of another LDP-polygon, and there exists $i$ with $1 \leqslant i \leqslant d$ such that $F_{i}$ and $F_{i+1}$ are nonsingular. Then $F_{j}$ is singular for every $j \in\{1, \ldots, d\} \backslash\{i, i+1\}$.

Proof. It is obvious for $d=3$. We may assume that $d \geqslant 4, i=2$ and

$$
v_{2}=\left(\begin{array}{c}
-1 \\
0
\end{array}\right), v_{3}=\left(\begin{array}{c}
0 \\
-1
\end{array}\right), v_{4}=\left(\begin{array}{l}
1 \\
a
\end{array}\right)
$$

for $a \in \mathbb{Z}$. To deduce a contradiction we assume that there exists $j \in\{1,4,5, \ldots, d\}$ such that $F_{j}$ is nonsingular. Proposition 7 and $f(3)=a+2$ imply $a \geqslant-1$. Since $Q$ is not a blow-up of a smaller LDP-polygon at a nonsingular edge, we have $v_{2}+v_{4} \neq v_{3}$. Thus, $a \geqslant 0$ and $y_{5}, \ldots, y_{d}, y_{1} \geqslant 1$. In particular, $\sigma_{2} \cup \sigma_{3} \supset\left\{\left(\begin{array}{c}x \\ y\end{array}\right) \in \mathbb{R}^{2} ; y \leqslant 0\right\}$.

Case 1. Suppose that $5 \leqslant j \leqslant d$. If $F_{j-1}\left(\operatorname{resp} . F_{j+1}\right)$ is nonsingular, then $a=0$ and $\sigma_{j-1} \cup \sigma_{j}$ (resp. $\sigma_{j} \cup \sigma_{j+1}$ ) coincides with $\left\{\left(\begin{array}{l}x \\ y\end{array}\right) \in \mathbb{R}^{2} ; y \geqslant 0\right\}$, a contradiction. Hence, both $F_{j-1}$ and $F_{j+1}$ are singular. However, by Lemma $10(1)$, the cones $\sigma_{j-1}, \sigma_{j}, \sigma_{j+1}$ cover more than a half-plane. This is a contradiction. 
Case 2. Suppose that $j=1$ or $j=4$. We may assume that $j=4$. Since $Q$ has at least one singular edge, we may further assume that $F_{1}$ is singular. Then $y_{1} \geqslant 2$ and $v_{5}=\left(\begin{array}{c}b \\ a b+1\end{array}\right)$ for some $b \in \mathbb{Z}$. Note that $a b=y_{5}-1 \geqslant 0$. Proposition 7 and $f(2)=x_{1}+y_{1}+1$ imply $x_{1} \geqslant-y_{1}$. Since $y_{1} \geqslant 2$ and $v_{1}$ is primitive, we have $x_{1} \geqslant 1-y_{1}$ and $x_{1} \neq 0$. Furthermore, $1 \leqslant f(4)=2-b$ and $v_{3}+v_{5} \neq v_{4}$ imply $b \leqslant 0$. There are three subcases to consider.

Subcase 2.1. Suppose that $x_{1} \geqslant 1$. Then $d \geqslant 5$ since $x_{5}=b \leqslant 0$. Obviously, $\operatorname{det}\left(v_{5}, v_{1}\right)=b y_{1}-(a b+1) x_{1} \geqslant 1$. On the other hand, if $a=0$, then $b y_{1} \leqslant 0$ and $-x_{1} \leqslant-1$, which means that $\operatorname{det}\left(v_{5}, v_{1}\right) \leqslant-1$. But even if $a \geqslant 1$, we have $a b \geqslant 0$ and $b \leqslant 0$, meaning that $b=0$, and again $\operatorname{det}\left(v_{5}, v_{1}\right) \leqslant-1$. This is a contradiction.

Subcase 2.2. Suppose that $x_{1} \leqslant-1$ and $a=0$. Then $v_{4}=\left(\begin{array}{l}1 \\ 0\end{array}\right), v_{5}=\left(\begin{array}{l}b \\ 1\end{array}\right)$ and $d \geqslant 5$. We see that $\left(\operatorname{det}\left(v_{5}, v_{1}\right)+1\right) / y_{1}>0$ and

$$
\frac{\operatorname{det}\left(v_{5}, v_{1}\right)+1}{y_{1}}\left(\left(1-\frac{1}{\operatorname{det}\left(v_{5}, v_{1}\right)+1}\right) v_{4}+\frac{1}{\operatorname{det}\left(v_{5}, v_{1}\right)+1} v_{1}\right)=v_{5} \text {. }
$$

However,

$$
\operatorname{det}\left(v_{5}, v_{1}\right)+1=b y_{1}-x_{1}+1 \leqslant b y_{1}+y_{1} \leqslant y_{1}
$$

and thus $0<\left(\operatorname{det}\left(v_{5}, v_{1}\right)+1\right) / y_{1} \leqslant 1$, which contradicts the fact that $v_{5}$ is a vertex of $Q$.

Subcase 2.3. Suppose that $x_{1} \leqslant-1$ and $a \geqslant 1$. Since $a b \geqslant 0$ and $b \leqslant 0$, we must have $b=0$. Hence $v_{4}=\left(\begin{array}{l}1 \\ a\end{array}\right), v_{5}=\left(\begin{array}{l}0 \\ 1\end{array}\right)$ and $d \geqslant 5$. We see that $\left(1-x_{1}\right) / \operatorname{det}\left(v_{4}, v_{1}\right)>0$ and

$$
\frac{1-x_{1}}{\operatorname{det}\left(v_{4}, v_{1}\right)}\left(\left(1-\frac{1}{1-x_{1}}\right) v_{4}+\frac{1}{1-x_{1}} v_{1}\right)=v_{5} \text {. }
$$

However,

$$
\operatorname{det}\left(v_{4}, v_{1}\right)=y_{1}-a x_{1} \geqslant\left(1-x_{1}\right)-a x_{1} \geqslant 1-x_{1}+a>1-x_{1}
$$

and thus $0<\left(1-x_{1}\right) / \operatorname{det}\left(v_{4}, v_{1}\right)<1$, which contradicts the fact that $v_{5}$ is a vertex of $Q$.

Thus, we have reached a contradiction in every case. Hence, $F_{1}, F_{4}, F_{5}, \ldots, F_{d}$ are singular.

We are now ready to prove Theorem 3 .

Proof of Theorem 3. Let $Q$ be an LDP-polygon with two singular edges. Then there exists at least one $i$ with $1 \leqslant i \leqslant d$ such that $F_{i}$ is nonsingular. Hence we may assume that $v_{1}=\left(\begin{array}{l}1 \\ 0\end{array}\right)$ and $v_{2}=\left(\begin{array}{l}0 \\ 1\end{array}\right)$.

The case where $d=3$. Since $|\operatorname{Sing}(Q)|=2$, the edges $F_{2}$ and $F_{3}$ are singular. Hence $v_{3}=\left(\begin{array}{l}-p \\ -q\end{array}\right)$ for some $p, q \in \mathbb{Z}$ with $p, q \geqslant 2$ and $\operatorname{gcd}(p, q)=1$. Conversely, for any 
$p, q \in \mathbb{Z}$ with $p, q \geqslant 2$ and $\operatorname{gcd}(p, q)=1$, the convex hull conv $\left\{\left(\begin{array}{l}1 \\ 0\end{array}\right),\left(\begin{array}{l}0 \\ 1\end{array}\right),\left(\begin{array}{l}-p \\ -q\end{array}\right)\right\}$ is an LDP-triangle with two singular edges.

The case where $d=4$. By Lemma $10(2)$, either $F_{2}$ or $F_{4}$ is nonsingular. We may assume that $F_{2}$ is nonsingular. Then $v_{3}=\left(\begin{array}{c}-1 \\ p\end{array}\right), v_{4}=\left(\begin{array}{c}q \\ r\end{array}\right)$ for some $p, q, r \in \mathbb{Z}$ with $\operatorname{gcd}(q, r)=1$. Hence, it suffices to show that for $p, q, r \in \mathbb{Z}$ with $\operatorname{gcd}(q, r)=1$, the sequence

$$
v_{1}=\left(\begin{array}{l}
1 \\
0
\end{array}\right), v_{2}=\left(\begin{array}{l}
0 \\
1
\end{array}\right), v_{3}=\left(\begin{array}{c}
-1 \\
p
\end{array}\right), v_{4}=\left(\begin{array}{l}
q \\
r
\end{array}\right)
$$

determines an LDP-polygon $Q=\operatorname{conv}\left\{v_{1}, \ldots, v_{4}\right\}$ (that is, $v_{1}, \ldots, v_{4}$ go counterclockwise around the origin exactly once in this order, and every $v_{i}$ is a vertex of $Q$ ) and $|\operatorname{Sing}(Q)|=$ 2 if and only if $p \leqslant 1$ and $r \leqslant \min \{-p q-2,-2,-q-1, q-p q-1\}$. We first observe that if the sequence determines $Q$, then we have

- $F_{1}$ and $F_{2}$ are nonsingular;

- $f(2) \geqslant 1 \Leftrightarrow p \leqslant 1$

- $F_{3}$ is singular if and only if $r \leqslant-p q-2$;

- $F_{4}$ is singular if and only if $r \leqslant-2$;

- $f(1) \geqslant 1 \Leftrightarrow r \leqslant-q$;

- $f(3) \geqslant 1 \Leftrightarrow r \leqslant q-p q$.

Suppose that the sequence determines $Q$ and $|\operatorname{Sing}(Q)|=2$. Since $r \leqslant-2$ and $v_{4}$ is primitive, we have $r \leqslant-q-1$. If $r=q-p q$, then $q= \pm 1$ since $v_{4}$ is primitive, which contradicts that $r \leqslant-p q-2$. Hence, $r \leqslant q-p q-1$. Therefore, $p \leqslant 1$ and $r \leqslant \min \{-p q-2,-2,-q-1, q-p q-1\}$.

Conversely, suppose that $p \leqslant 1$ and $r \leqslant \min \{-p q-2,-2,-q-1, q-p q-1\}$. It suffices to show that $f(4) \geqslant 1$, that is, $p(1-q)-2 r \geqslant 1$. Since $r \leqslant-2$ and $v_{4}$ is primitive, we have $q \neq 0$.

Case 1. Suppose $q \geqslant 1$. Then $p \leqslant 1$ implies $p(1-q) \geqslant 1-q$. Since $r \leqslant-q-1$, we have $-2 r \geqslant 2 q+2$. Thus,

$$
f(4)=p(1-q)-2 r \geqslant(1-q)+(2 q+2)=q+3 \geqslant 4 .
$$

Case 2. Suppose $q \leqslant-1$. It suffices to show $p q(1-q)-2 q r \leqslant q$. The assumption $p q \leqslant-r-2$ implies $p q(1-q) \leqslant(q-1)(r+2)$. Since $r+2 \leqslant 0$ and $q-1 \geqslant 2 q$, we have $(q-1)(r+2) \leqslant 2 q(r+2)$. Thus,

$$
p q(1-q)-2 q r \leqslant 2 q(r+2)-2 q r=4 q<q .
$$

In every case, we obtain $f(4) \geqslant 1$. Therefore, $Q=\operatorname{conv}\left\{v_{1}, \ldots, v_{4}\right\}$ is an LDPquadrilateral with $|\operatorname{Sing}(Q)|=2$.

The case where $d \geqslant 5$. First we show the following claims: 
Claim 12. Every LDP-polygon $Q$ with exactly two singular edges and more than four edges in total is an iterated blow-up, at nonsingular edges, of an LDP-quadrilateral.

Proof of Claim 12. We use induction on $d$. Assume $d \geqslant 5$. We may assume that $F_{1}$ and $F_{2}$ are nonsingular. If $Q=Q_{d}$ cannot be obtained by blowing up a nonsingular edge of another LDP-polygon, then $F_{3}, \ldots, F_{d}$ are all singular by Lemma 11, which contradicts that $|\operatorname{Sing}(Q)|=2$. Hence, $Q_{d}$ is the blow-up of a smaller LDP-polygon at a nonsingular edge. We may further assume that $v_{2}=v_{1}+v_{3}$. Let $Q_{d-1}=\operatorname{conv}\left\{v_{1}, v_{3}, v_{4}, \ldots, v_{d}\right\}$. Then $Q_{d}$ is the blow-up of $Q_{d-1}$ at the nonsingular edge $v_{1} v_{3}$. By induction hypothesis there is a sequence of LDP-polygons

$$
Q=Q_{d}, Q_{d-1}, \ldots, Q_{4},
$$

where $Q_{i}$ is the blow-up of $Q_{i-1}$ at a nonsingular edge for $5 \leqslant i \leqslant d$.

Claim 13. Every LDP-polygon with exactly two singular edges has five edges or less in total.

Proof of Claim 13. Let $Q^{\prime}$ be an LDP-quadrilateral with $\left|\operatorname{Sing}\left(Q^{\prime}\right)\right|=2$, and let $Q=$ $\operatorname{conv}\left\{v_{1}, \ldots, v_{5}\right\}$ be the blow-up of $Q^{\prime}$ at one nonsingular edge. Then we may assume that

$$
v_{1}=\left(\begin{array}{l}
1 \\
0
\end{array}\right), v_{2}=\left(\begin{array}{l}
0 \\
1
\end{array}\right), v_{3}=\left(\begin{array}{c}
-1 \\
p+1
\end{array}\right), v_{4}=\left(\begin{array}{c}
-1 \\
p
\end{array}\right), v_{5}=\left(\begin{array}{l}
q \\
r
\end{array}\right)
$$

for $p, q, r \in \mathbb{Z}$ with

$$
p \leqslant 1, r \leqslant \min \{-p q-2,-2,-q-1, q-p q-1\}, \operatorname{gcd}(q, r)=1 .
$$

By Claim 12, it suffices to show that the blow-up of $Q$ at any nonsingular edge does not increase the number of vertices. Proposition 7 and $f(4)=q+1$ imply $q \geqslant 0$. Since $r \leqslant-2$ and $v_{5}$ is primitive, we have $q \geqslant 1$. The nonsingular edges of $Q$ are $F_{1}, F_{2}, F_{3}$. However, we see that

$$
\begin{aligned}
& \operatorname{det}\left(v_{5}, v_{1}\right)+\operatorname{det}\left(v_{1}, v_{1}+v_{2}\right)+\operatorname{det}\left(v_{1}+v_{2}, v_{5}\right)=1-q \leqslant 0, \\
& \operatorname{det}\left(v_{2}+v_{3}, v_{3}\right)+\operatorname{det}\left(v_{3}, v_{4}\right)+\operatorname{det}\left(v_{4}, v_{2}+v_{3}\right)=0, \\
& \operatorname{det}\left(v_{2}, v_{3}\right)+\operatorname{det}\left(v_{3}, v_{3}+v_{4}\right)+\operatorname{det}\left(v_{3}+v_{4}, v_{2}\right)=0 .
\end{aligned}
$$

Hence, the blow-up of $Q$ at any nonsingular edge does not increase the number of vertices. This completes the proof of Claim 13.

By Claims 12 and 13, it suffices to show that for $p, q, r \in \mathbb{Z}$ with $\operatorname{gcd}(q, r)=1$, the sequence

$$
v_{1}=\left(\begin{array}{l}
1 \\
0
\end{array}\right), v_{2}=\left(\begin{array}{l}
0 \\
1
\end{array}\right), v_{3}=\left(\begin{array}{c}
-1 \\
p+1
\end{array}\right), v_{4}=\left(\begin{array}{c}
-1 \\
p
\end{array}\right), v_{5}=\left(\begin{array}{l}
q \\
r
\end{array}\right)
$$

determines an LDP-polygon $Q=\operatorname{conv}\left\{v_{1}, \ldots, v_{5}\right\}$ and $|\operatorname{Sing}(Q)|=2$ if and only if $p \leqslant 0$ and $1 \leqslant q \leqslant-r-1$. We observe that if the sequence determines $Q$, then we have 
- $F_{1}, F_{2}, F_{3}$ are nonsingular;

- $F_{5}$ is singular if and only if $r \leqslant-2$;

- $f(1) \geqslant 1 \Leftrightarrow q \leqslant-r$;

- $f(2) \geqslant 1 \Leftrightarrow p \leqslant 0$

- $f(3)=1$;

- $f(4) \geqslant 1 \Leftrightarrow q \geqslant 0$.

If the sequence determines $Q$ and $|\operatorname{Sing}(Q)|=2$, then $p \leqslant 0$ and $1 \leqslant q \leqslant-r-1$, since $r \leqslant-2$ and $v_{5}$ is primitive. Conversely, suppose that $p \leqslant 0$ and $1 \leqslant q \leqslant-r-1$. We need to show that $f(5) \geqslant 1$ and $\operatorname{det}\left(v_{4}, v_{5}\right) \geqslant 2$. Since $p(1-q) \geqslant 0$ and $r \leqslant-2$, we have $f(5)=p(1-q)-2 r \geqslant 4$ and $\operatorname{det}\left(v_{4}, v_{5}\right)=-r-p q \geqslant 2$. Thus, $Q=\operatorname{conv}\left\{v_{1}, \ldots, v_{5}\right\}$ is an LDP-pentagon with $|\operatorname{Sing}(Q)|=2$. This completes the proof of Theorem 3 .

\section{Proof of Theorem 4}

In this section, we prove Theorem 4. First we show the following lemma:

Lemma 14. If $Q$ is an LDP-pentagon with exactly two nonsingular edges, then these two edges are consecutive.

Proof. To deduce a contradiction we assume that there is an LDP-pentagon $Q$ with $\operatorname{Sing}(Q)=\left\{F_{1}, F_{3}, F_{5}\right\}$. We may assume that $v_{2}=\left(\begin{array}{c}-1 \\ 0\end{array}\right), v_{3}=\left(\begin{array}{c}0 \\ -1\end{array}\right)$. Then $y_{1} \geqslant 2, x_{4} \geqslant 2, x_{1}+y_{1} \geqslant 1, x_{4}+y_{4} \geqslant 1$.

Case 1. Suppose $y_{5} \leqslant 0$. Then $x_{5} \geqslant 1$. We will show that $x_{5}+y_{5}-x_{4}-y_{4} \geqslant 1$. Since $f(5)=\left(x_{5}-x_{4}\right) y_{1}-x_{1}\left(y_{5}-y_{4}\right)+1$ and $f(4)=x_{4}-x_{5}+1$, Proposition 7 gives $\left(x_{5}-x_{4}\right) y_{1}-x_{1}\left(y_{5}-y_{4}\right) \geqslant 0$ and $x_{4} \geqslant x_{5}$. It follows that

$$
1=\operatorname{det}\left(v_{4}, v_{5}\right)=x_{4} y_{5}-x_{5} y_{4} \leqslant x_{5} y_{5}-x_{5} y_{4}=x_{5}\left(y_{5}-y_{4}\right)
$$

and thus $y_{5}-y_{4} \geqslant 1$. Since $x_{1} \geqslant 1-y_{1}$, we have $x_{1}\left(y_{5}-y_{4}\right) \geqslant\left(1-y_{1}\right)\left(y_{5}-y_{4}\right)$. Hence,

$$
\begin{aligned}
0 & \leqslant\left(x_{5}-x_{4}\right) y_{1}-x_{1}\left(y_{5}-y_{4}\right) \\
& \leqslant\left(x_{5}-x_{4}\right) y_{1}-\left(1-y_{1}\right)\left(y_{5}-y_{4}\right) \\
& =\left(x_{5}+y_{5}-x_{4}-y_{4}\right) y_{1}-\left(y_{5}-y_{4}\right) \\
& \leqslant\left(x_{5}+y_{5}-x_{4}-y_{4}\right) y_{1}-1 .
\end{aligned}
$$

Therefore, $x_{5}+y_{5}-x_{4}-y_{4} \geqslant 1$.

Let $v^{\prime}=\left(\begin{array}{c}-1 \\ 1\end{array}\right)$. We calculate

$$
\operatorname{det}\left(v_{5}, v^{\prime}\right)+\operatorname{det}\left(v^{\prime}, v_{3}\right)+\operatorname{det}\left(v_{3}, v_{5}\right)=\left(x_{5}+y_{5}\right)+1+x_{5}
$$




$$
\begin{aligned}
& \geqslant\left(x_{4}+y_{4}+1\right)+1+x_{5} \geqslant 3+x_{5} \geqslant 4, \\
\operatorname{det}\left(v^{\prime}, v_{3}\right)+\operatorname{det}\left(v_{3}, v_{4}\right)+\operatorname{det}\left(v_{4}, v^{\prime}\right) & =1+x_{4}+\left(x_{4}+y_{4}\right) \geqslant 1+x_{4}+1 \geqslant 4, \\
\operatorname{det}\left(v_{3}, v_{4}\right)+\operatorname{det}\left(v_{4}, v_{5}\right)+\operatorname{det}\left(v_{5}, v_{3}\right) & =f(4) \geqslant 1, \\
\operatorname{det}\left(v_{4}, v_{5}\right)+\operatorname{det}\left(v_{5}, v^{\prime}\right)+\operatorname{det}\left(v^{\prime}, v_{4}\right) & =1+x_{5}+y_{5}-x_{4}-y_{4} \geqslant 2 .
\end{aligned}
$$

Hence, $\operatorname{conv}\left\{v^{\prime}, v_{3}, v_{4}, v_{5}\right\}$ is an LDP-quadrilateral. However, $\operatorname{det}\left(v_{3}, v_{4}\right) \neq 1$ and

$$
\operatorname{det}\left(v_{5}, v^{\prime}\right)=x_{5}+y_{5} \geqslant x_{4}+y_{4}+1 \geqslant 2
$$

while $\operatorname{det}\left(v^{\prime}, v_{3}\right)=\operatorname{det}\left(v_{4}, v_{5}\right)=1$, which contradicts Theorem 3 .

Case 2. Suppose $y_{5}=1$. Then $Q^{\prime}=\operatorname{conv}\left\{v_{2}, v_{3}, v_{4}, v_{5}\right\}$ is an LDP-quadrilateral with $\left|\operatorname{Sing}\left(Q^{\prime}\right)\right|=1$. By Theorem 2, we have either $v_{2}=v_{5}+v_{3}$ or $v_{5}=v_{4}+v_{2}$. If $v_{2}=v_{5}+v_{3}$, then $v_{5}=v_{2}-v_{3}=\left(\begin{array}{c}-1 \\ 1\end{array}\right)$ and thus $2 \leqslant \operatorname{det}\left(v_{5}, v_{1}\right)=-x_{1}-y_{1} \leqslant-1$, a contradiction. If $v_{5}=v_{4}+v_{2}$, then $v_{4}=v_{5}-v_{2}=\left(\begin{array}{c}x_{5}+1 \\ 1\end{array}\right)$ and thus

$$
1 \leqslant \operatorname{det}\left(v_{4}, v_{5}\right)+\operatorname{det}\left(v_{5}, v_{1}\right)+\operatorname{det}\left(v_{1}, v_{4}\right)=1-y_{1} \leqslant 1-2=-1,
$$

a contradiction.

Case 3. Suppose $y_{5} \geqslant 2$. Then $Q^{\prime}=\operatorname{conv}\left\{v_{2}, v_{3}, v_{4}, v_{5}\right\}$ is an LDP-quadrilateral with $\left|\operatorname{Sing}\left(Q^{\prime}\right)\right|=2$. However, $\operatorname{det}\left(v_{3}, v_{4}\right) \neq 1$ and $\operatorname{det}\left(v_{5}, v_{2}\right)=y_{5} \geqslant 2$ while $\operatorname{det}\left(v_{2}, v_{3}\right)=$ $\operatorname{det}\left(v_{4}, v_{5}\right)=1$, which contradicts Theorem 3 .

Thus, we have reached a contradiction in every case.

Proof of Theorem 4. Let $Q$ be an LDP-polygon with three singular edges. We may assume that $v_{1}=\left(\begin{array}{l}1 \\ 0\end{array}\right)$.

The case where $d=3$. Since $|\operatorname{Sing}(Q)|=3$, all edges of $Q$ are singular. Hence $v_{2}=\left(\begin{array}{c}p \\ q\end{array}\right)$ and $v_{3}=\left(\begin{array}{c}r \\ s\end{array}\right)$ for some $p, q, r, s \in \mathbb{Z}$ with $q \geqslant 2, s \leqslant-2, p s-q r \geqslant 2$ and $\operatorname{gcd}(p, q)=\operatorname{gcd}(r, s)=1$. Conversely, for any $p, q, r, s \in \mathbb{Z}$ with $q \geqslant 2, s \leqslant-2, p s-q r \geqslant 2$ and $\operatorname{gcd}(p, q)=\operatorname{gcd}(r, s)=1$, the convex hull conv $\left\{\left(\begin{array}{l}1 \\ 0\end{array}\right),\left(\begin{array}{c}p \\ q\end{array}\right),\left(\begin{array}{l}r \\ s\end{array}\right)\right\}$ is an LDPtriangle with three singular edges.

The case where $d=4$. We may assume that $v_{2}=\left(\begin{array}{l}0 \\ 1\end{array}\right)$ and $F_{2}, F_{3}, F_{4}$ are singular. Then $v_{3}=\left(\begin{array}{c}p \\ q\end{array}\right)$ and $v_{4}=\left(\begin{array}{c}r \\ s\end{array}\right)$ for some $p, q, r, s \in \mathbb{Z}$ with $\operatorname{gcd}(p, q)=\operatorname{gcd}(r, s)=1$. We observe the following:

- $F_{2}$ is singular if and only if $p \leqslant-2$;

- $f(2) \geqslant 1 \Leftrightarrow p \leqslant-q$;

- $F_{4}$ is singular if and only if $s \leqslant-2$; 
- $f(1) \geqslant 1 \Leftrightarrow s \leqslant-r$;

- $F_{3}$ is singular if and only if $p s-q r \geqslant 2$; moreover, this inequality together with $p, s \leqslant-2$ guarantees that the four rays $(1,0),(0,1),(p, q)$ and $(r, s)$ are in counterclockwise order.

- $f(3) \geqslant 1 \Leftrightarrow p s-q r \geqslant p-r+1$

- $f(4) \geqslant 1 \Leftrightarrow p s-q r \geqslant s-q+1$.

Hence, the assertion holds.

The case where $d \geqslant 5$. Firstly, we assume that $d=5$. By Lemma 14 , there exists $i$ such that $F_{i}$ and $F_{i+1}$ are nonsingular. We may assume that $i=1, v_{2}=\left(\begin{array}{l}0 \\ 1\end{array}\right)$. Then

$$
v_{3}=\left(\begin{array}{c}
-1 \\
p
\end{array}\right), v_{4}=\left(\begin{array}{c}
-q \\
p q-r
\end{array}\right), v_{5}=\left(\begin{array}{c}
s \\
-t
\end{array}\right)
$$

for some $p, q, r, s, t \in \mathbb{Z}$ with $\operatorname{gcd}(q, r)=\operatorname{gcd}(s, t)=1$. Hence, it suffices to show that for $p, q, r, s, t \in \mathbb{Z}$ with $\operatorname{gcd}(q, r)=\operatorname{gcd}(s, t)=1$, the sequence

$$
v_{1}=\left(\begin{array}{l}
1 \\
0
\end{array}\right), v_{2}=\left(\begin{array}{c}
0 \\
1
\end{array}\right), v_{3}=\left(\begin{array}{c}
-1 \\
p
\end{array}\right), v_{4}=\left(\begin{array}{c}
-q \\
p q-r
\end{array}\right), v_{5}=\left(\begin{array}{c}
s \\
-t
\end{array}\right)
$$

determines an LDP-polygon $Q=\operatorname{conv}\left\{v_{1}, \ldots, v_{5}\right\}$ and $|\operatorname{Sing}(Q)|=3$ if and only if

$$
\begin{aligned}
p & \leqslant 1, \\
r & \geqslant \max \{2, q+1\}, \\
t & \geqslant \max \{2, s+1\}, \\
q t+r s-p q s & \geqslant \max \{2, t-r-p s+1, r-t-p q+1\}, \\
p q-r & \leqslant-1 .
\end{aligned}
$$

If the above sequence determines $Q$, then we observe the following:

- $F_{1}$ and $F_{2}$ are nonsingular;

- $f(2) \geqslant 1 \Leftrightarrow p \leqslant 1$

- $F_{3}$ is singular if and only if $r \geqslant 2$;

- $f(3) \geqslant 1 \Leftrightarrow r \geqslant q$

- $F_{5}$ is singular if and only if $t \geqslant 2$;

- $f(1) \geqslant 1 \Leftrightarrow t \geqslant s$;

- $F_{4}$ is singular if and only if $q t+r s-p q s \geqslant 2$; 
- $f(4) \geqslant 1 \Leftrightarrow q t+r s-p q s \geqslant t-r-p s+1$;

- $f(5) \geqslant 1 \Leftrightarrow q t+r s-p q s \geqslant r-t-p q+1$.

Suppose that the same sequence determines $Q$ and $|\operatorname{Sing}(Q)|=3$. Since $t \geqslant 2$ and $v_{5}$ is primitive, we have $t \geqslant s+1$. If $r=q$, then $q= \pm 1$ since $v_{4}$ is primitive, which contradicts that $r \geqslant 2$. Hence, $r \geqslant q+1$. It remains to show that $p q-r \leqslant-1$. If $p \leqslant 0$, then $p q-r \leqslant-1$ holds obviously. If $p=1$, then $p q-r=q-r \leqslant q-(q+1)=-1$. We therefore obtain the required inequalities. The converse is obvious.

To prove the remaining part of the theorem, we need the following claim:

Claim 15. Every LDP-polygon $Q$ with three singular edges and more than five edges in total is an iterated blow-up, at nonsingular edges, of an LDP-pentagon.

Proof of Claim 15. We use induction on $d$. Assume $d=6$. To deduce a contradiction we assume that $Q$ cannot be obtained by blowing up a nonsingular edge of another LDPpolygon. By Lemma 11, if $F_{i}$ is nonsingular, then both $F_{i-1}$ and $F_{i+1}$ are singular. We may assume that $\operatorname{Sing}(Q)=\left\{F_{1}, F_{3}, F_{5}\right\}$. By Lemma $10(1)$, we have $\operatorname{det}\left(v_{4}, v_{1}\right) \geqslant 2$. We consider the LDP-pentagon $\operatorname{conv}\left\{v_{1}, \ldots, v_{5}\right\}$. If $\operatorname{det}\left(v_{5}, v_{1}\right)=1$, then this contradicts Theorem 3. Otherwise this contradicts Lemma 14. Hence, $Q$ is the blow-up of a smaller LDP-polygon at a nonsingular edge.

In the case $d \geqslant 7$, we may assume that $F_{1}$ and $F_{2}$ are nonsingular. The rest of the proof is as for Claim 12 .

Let $Q^{\prime}$ be an LDP-pentagon with $\left|\operatorname{Sing}\left(Q^{\prime}\right)\right|=3$, and let $Q=\operatorname{conv}\left\{v_{1}, \ldots, v_{6}\right\}$ be the blow-up of $Q^{\prime}$ at one nonsingular edge. Then we may assume that

$$
\begin{aligned}
& v_{1}=\left(\begin{array}{l}
1 \\
0
\end{array}\right), v_{2}=\left(\begin{array}{l}
0 \\
1
\end{array}\right), v_{3}=\left(\begin{array}{c}
-1 \\
p+1
\end{array}\right), \\
& v_{4}=\left(\begin{array}{c}
-1 \\
p
\end{array}\right), v_{5}=\left(\begin{array}{c}
-q \\
p q-r
\end{array}\right), v_{6}=\left(\begin{array}{c}
s \\
-t
\end{array}\right)
\end{aligned}
$$

for $p, q, r, s, t \in \mathbb{Z}$ with

$$
\begin{aligned}
p & \leqslant 1, \\
r & \geqslant \max \{2, q+1\}, \\
t & \geqslant \max \{2, s+1\}, \\
q t+r s-p q s & \geqslant \max \{2, t-r-p s+1, r-t-p q+1\}, \\
p q-r & \leqslant-1
\end{aligned}
$$

and $\operatorname{gcd}(q, r)=\operatorname{gcd}(s, t)=1$. By Claim 15, to prove the remaining part it suffices to show that the blow-up of $Q$ at any nonsingular edge does not increase the number of vertices. Proposition 7 and $f(4)=1-q$ imply $q \leqslant 0$. Since $r-p q \geqslant 1$ and $(r-p q) s=r s-p q s \geqslant$ $2-q t \geqslant 2$, we have $s \geqslant 1$. The nonsingular edges of $Q$ are $F_{1}, F_{2}, F_{3}$. However, we see that

$$
\operatorname{det}\left(v_{6}, v_{1}\right)+\operatorname{det}\left(v_{1}, v_{1}+v_{2}\right)+\operatorname{det}\left(v_{1}+v_{2}, v_{6}\right)=1-s \leqslant 0
$$




$$
\begin{aligned}
& \operatorname{det}\left(v_{2}+v_{3}, v_{3}\right)+\operatorname{det}\left(v_{3}, v_{4}\right)+\operatorname{det}\left(v_{4}, v_{2}+v_{3}\right)=0, \\
& \operatorname{det}\left(v_{2}, v_{3}\right)+\operatorname{det}\left(v_{3}, v_{3}+v_{4}\right)+\operatorname{det}\left(v_{3}+v_{4}, v_{2}\right)=0 .
\end{aligned}
$$

Hence, the blow-up of $Q$ at any nonsingular edge does not increase the number of vertices. This completes the proof of Theorem 4 .

The following proposition holds even for $|\operatorname{Sing}(Q)| \geqslant 4$.

Proposition 16. In every LDP-polygon $Q$ the nonsingular edges (hence also all the singular edges) form a consecutive sequence.

Proof. We may assume that $Q$ cannot be obtained by blowing up a nonsingular edge of another LDP-polygon. If $d \leqslant 5$, then the assertion follows from Theorems 3 and 4 .

Assume $d=6$. If $|\operatorname{Sing}(Q)| \geqslant 5$, then the assertion is obvious. If $|\operatorname{Sing}(Q)| \leqslant 3$, then the assertion follows from Theorems 3 and 4. Hence, we may further assume that $|\operatorname{Sing}(Q)|=4$ and $F_{2}$ is nonsingular. To deduce a contradiction we assume that the other nonsingular edge is one of $F_{4}, F_{5}, F_{6}$.

Case 1. Suppose that either $F_{4}$ or $F_{6}$ is nonsingular. We may assume that $F_{4}$ is nonsingular. By Lemma $10(1)$, we have $\operatorname{det}\left(v_{4}, v_{1}\right) \geqslant 2$. We consider the LDP-pentagon $\operatorname{conv}\left\{v_{1}, \ldots, v_{5}\right\}$. If $\operatorname{det}\left(v_{5}, v_{1}\right)=1$, then this contradicts Theorem 3. Otherwise this contradicts Theorem 4.

Case 2. Suppose that $F_{5}$ is nonsingular. By Lemma $10(1)$, the cones $\sigma_{1}, \sigma_{2}, \sigma_{3}$ cover more than a half-plane. Similarly, $\sigma_{4}, \sigma_{5}, \sigma_{6}$ cover more than a half-plane. This is a contradiction.

Hence, the other nonsingular edge is either $F_{1}$ or $F_{3}$. Therefore, the assertion holds for $d=6$.

We prove the assertion for $d \geqslant 7$. We use induction on $d$. If there exists $i$ such that $F_{i}$ and $F_{i+1}$ are nonsingular, then the remaining edges are all singular by Lemma 11 . Hence, we may assume that if $F_{i}$ is nonsingular, then both $F_{i-1}$ and $F_{i+1}$ are singular. To deduce a contradiction we assume that there are $i$ and $j$ with $1 \leqslant i<j \leqslant d$ and $j \geqslant i+2$ such that $F_{i}$ and $F_{j}$ are nonsingular. By Lemma 10 (1), we must have

$$
(i, j) \in\{(1,3),(2,4), \ldots,(d-2, d),(1, d-1),(2, d)\} .
$$

We may further assume that $(i, j)=(2,4)$. Then $\operatorname{det}\left(v_{4}, v_{1}\right) \geqslant 2$ by Lemma $10(1)$. We consider the LDP-polygon conv $\left\{v_{1}, \ldots, v_{d-1}\right\}$. We have $\operatorname{det}\left(v_{1}, v_{2}\right) \neq 1$ and $\operatorname{det}\left(v_{3}, v_{4}\right) \neq 1$ while $\operatorname{det}\left(v_{2}, v_{3}\right)=\operatorname{det}\left(v_{4}, v_{5}\right)=1$, which contradicts the hypothesis of induction. Hence, $|\operatorname{Sing}(Q)|=d-1$. Therefore, the assertion holds for $d$. This completes the proof.

Finally, we give an upper bound for the number of nonsingular edges of an LDPpolygon.

Corollary 17. Let $Q$ be an LDP-polygon. If $|\operatorname{Sing}(Q)| \geqslant 2$, then $Q$ has at most three nonsingular edges. 
Proof. By Proposition 16, we may assume that there exists an integer $n$ with $0 \leqslant n \leqslant d-2$ such that $F_{i}$ is nonsingular for $1 \leqslant i \leqslant n$ and $F_{i}$ is singular for $n+1 \leqslant i \leqslant d$. To deduce a contradiction we assume that $n \geqslant 4$. We may further assume that $v_{1}=\left(\begin{array}{l}1 \\ 0\end{array}\right)$ and $v_{2}=\left(\begin{array}{l}0 \\ 1\end{array}\right)$. We consider the LDP-polygon $Q^{\prime}=\operatorname{conv}\left\{v_{1}, \ldots, v_{n}, v_{n+1}, v_{d}\right\}$. Then $Q^{\prime}$ has more than five vertices. Since $y_{5}, y_{6}, \ldots, y_{n+1}, y_{d} \leqslant-1$, we have $\operatorname{det}\left(v_{n+1}, v_{d}\right) \geqslant 1$. If $\operatorname{det}\left(v_{n+1}, v_{d}\right)=1$, then $Q^{\prime}$ has exactly one singular edge, which contradicts Theorem 2 . Otherwise $Q^{\prime}$ has exactly two singular edges, which contradicts Theorem 3. Hence, we must have $n \leqslant 3$. This completes the proof.

\section{Acknowledgements}

The author would like to thank the editor and referee for their suggestions and comments which have greatly improved the manuscript. He would also like to thank Benjamin Nill for his suggestion of Corollary 17.

\section{References}

[1] V. Alexeev and V. V. Nikulin, Del Pezzo and K3 surfaces, MSJ Memoirs, Vol. 15, Math. Soc. Japan, 2006.

[2] G. Belousov, Del Pezzo surfaces with log terminal singularities, Math. Notes 83 (2008), 152-161.

[3] G. Belousov, The maximal number of singular points on log del Pezzo surfaces, J. Math. Sci. Univ. Tokyo 16 (2009), 231-238.

[4] D. A. Cox, J. B. Little and H. K. Schenck, Toric Varieties, Graduate Studies in Mathematics, Vol. 124. American Mathematical Society, Providence, RI, 2011.

[5] D. I. Dais, Geometric combinatorics in the study of compact toric surfaces, In "Algebraic and Geometric Combinatorics" (edited by C. Athanasiadis et al.), Contemporary Mathematics, Vol. 423, American Mathematical Society, 2007, pp. 71-123.

[6] D. I. Dais, Classification of toric log del Pezzo surfaces having Picard number 1 and index $\leqslant 3$, Result. Math. 54 (2009), 219-252.

[7] D. I. Dais, Toric log del Pezzo surfaces with one singularity, Adv. Geom. 20 (2020), $121-138$.

[8] D. I. Dais and B. Nill, A boundedness result for toric log del Pezzo surfaces, Arch. Math. 91 (2008), 526-535.

[9] K. Fujita and K. Yasutake, Classification of log del Pezzo surfaces of index three, J. Math. Soc. Japan 69 (2017), 163-225.

[10] A. M. Kasprzyk, M. Kreuzer and B. Nill, On the combinatorial classification of toric log del Pezzo surfaces, LMS J. Comput. Math. 13 (2010), 33-46. 
[11] H. Kojima, Logarithmic del Pezzo surfaces of rank one with unique singular points, Japan J. Math. 25 (1999), 343-374.

[12] N. Nakayama, Classification of log del Pezzo surfaces of index two, J. Math. Sci. Univ. Tokyo 14 (2007), 293-498.

[13] V. V. Nikulin, Del Pezzo surfaces with log-terminal singularities I, Math. USSR-Sb. 66 (1990), 231-248; translation from Mat. Sb. 180 (1989), 226-243.

[14] V. V. Nikulin, Del Pezzo surfaces with log-terminal singularities II, Math. USSR-Izv. 33 (1989), 355-372; translation from Izv. Akad. Nauk SSSR 52 (1988), 1032-1050.

[15] V. V. Nikulin, Del Pezzo surfaces with log-terminal singularities III, Math. USSR-Izv. 35 (1990), 657-675; translation from Izv. Akad. Nauk SSSR 53 (1989), 1316-1334. 